\title{
MILLIARCSECOND STRUCTURES IN GRAVITATIONALLY LENSED SYSTEMS
}

\author{
ALOK R. PATNAIK AND RICHARD W. PORCAS \\ Max-Planck-Institut für Radioastronomie, \\ Auf dem Hügel 69, D-53121 Bonn, Germany.
}

\begin{abstract}
VLBI studies of small-scale structures in gravitationally lensed systems can provide important constraints on the lens mass distribution. We review the current status of VLBI observations of a number of lensed systems.
\end{abstract}

\section{Introduction}

Angular resolutions down to sub-mas scales are routinely achieved at radio wavelengths using the technique of Very Long Baseline Interferometry (VLBI). Although the wide fields of lens systems create special problems for VLBI (Porcas 1994; Garrett et al. 1994b), there are many areas where it can make a unique contribution:

1) Searching for new systems in the separation range 1-500 mas (see the paper by Patnaik et al. in these proceedings).

2) Confirming lens candidates, especially those without image redshift measurements, by revealing matching source structure in the images.

3) Measuring the time delay from structural variations in the images.

4) Investigation of the lens mass distribution by determining the relative magnification matrix between images and changes across extended images.

5) Looking for 'granularity' in the mass distribution (e.g. milli-lensing due to $10^{6} \mathrm{M}_{\odot}$ black holes) by detailed comparison of different images (see the paper by Garrett et al. in these proceedings).

6) Studying the lensing process itself by examining the surface brightness of resolved features and wavelength-dependent flux ratios between images. 7) Studying the imaged source under a magnifying glass, and with multiple versions of the same source (e.g. this can lead to a unique registration of maps made at different frequencies and epochs, Porcas and Patnaik 1995). 


\section{Review of individual lens systems}

0957+561 The earliest observations (Porcas et al. 1979, 1981; Haschick et al. 1981; Gorenstein et al. 1984, 1988) established the existence of core-jet structures in both $\mathrm{A}$ and $\mathrm{B}$ images and resulted in a relative magnification matrix used in subsequent modeling of the complex galaxy/cluster mass distribution. Recently, Garrett et al. (1994a) and Campbell et al. (1994) have used the extended jet structures to determine the magnification gradient over the images. Campbell et al. $(1994,1995)$ have also attempted to measure structural changes in both image cores; although no separation changes could be detected, a rough (but independent!) estimate of ca. 1 yr. for the time delay was determined from core flux density changes. The weak radio component $\mathrm{G}$, which may be emission from the galaxy $\mathrm{G} 1$ or a possible third image of the quasar, was first detected with VLBI by Gorenstein et al. (1983), and has also been observed on the EVN at $1.7 \mathrm{GHz}$, where it is unresolved (Garrett 1990). Higher resolution 2.3GHz observations (Rogers et al. 1989) indicate a possible core-jet structure for $\mathrm{G}$, with size ca. 7 mas. 2016+112 Heflin et al. (1991) have studied this system, which comprises 2 image components $\mathrm{A}, \mathrm{B}$ and a third component $\mathrm{C}$. At $1.7 \mathrm{GHz}$ with 3 mas resolution $\mathrm{A}$ and $\mathrm{B}$ must each be modeled with at least 2 sub-components, although apparently not easily related by a magnification matrix. Garrett et al. (these proceedings) have also mapped this system at $1.7 \mathrm{GHz}$, using the EVN. Component $\mathrm{C}$ breaks up into 4 sub-components: a colinear triple (C11, C12, C13) and a separated component, C2, which may be a third image, the radio counterpart to the source of Lyman $\alpha$ emission near C. Global $1.7 \mathrm{GHz}$ observations show that $\mathrm{C} 12, \mathrm{C} 13$ and $\mathrm{C} 2$ are compact on the 5mas scale (Garrett et al. 1994b).

MG0414+0534 This 'quad' comprises two bright, close components (A1, $\mathrm{A} 2$ ) and weaker components $\mathrm{B}$ and $\mathrm{C}$ (Hewitt et al. 1992). Our $1.7 \mathrm{GHz}$ EVN full-field map of this system is shown in Fig. 1 with a resolution of 25mas. Images A1, A2 and B are clearly resolved into subcomponents, with a bright 'core' and fainter extended regions. Component $\mathrm{C}$ is extended in the $\mathrm{N}$-S direction. In our higher resolution $5 \mathrm{GHz}$ EVN maps, the cores become more pronounced, and image $\mathrm{C}$ is also resolved into a core and secondary component. Unpublished global observations at $5 \mathrm{GHz}$ (Hewitt, private communication) show similar structures in the images.

PKS1830-211 This system contains two compact image components (NE, SW) separated by 1", embedded in an elliptical ring structure. No optical identification of lens or background object exists for this low Galactic latitude object. Southern Hemisphere VLBI observations (Jauncey et al. 1991) showed compact structure in both image components. Our $1.7 \mathrm{GHz}$ EVN map is shown in Fig. 2(left) with a resolution of 30mas. The NE compo- 


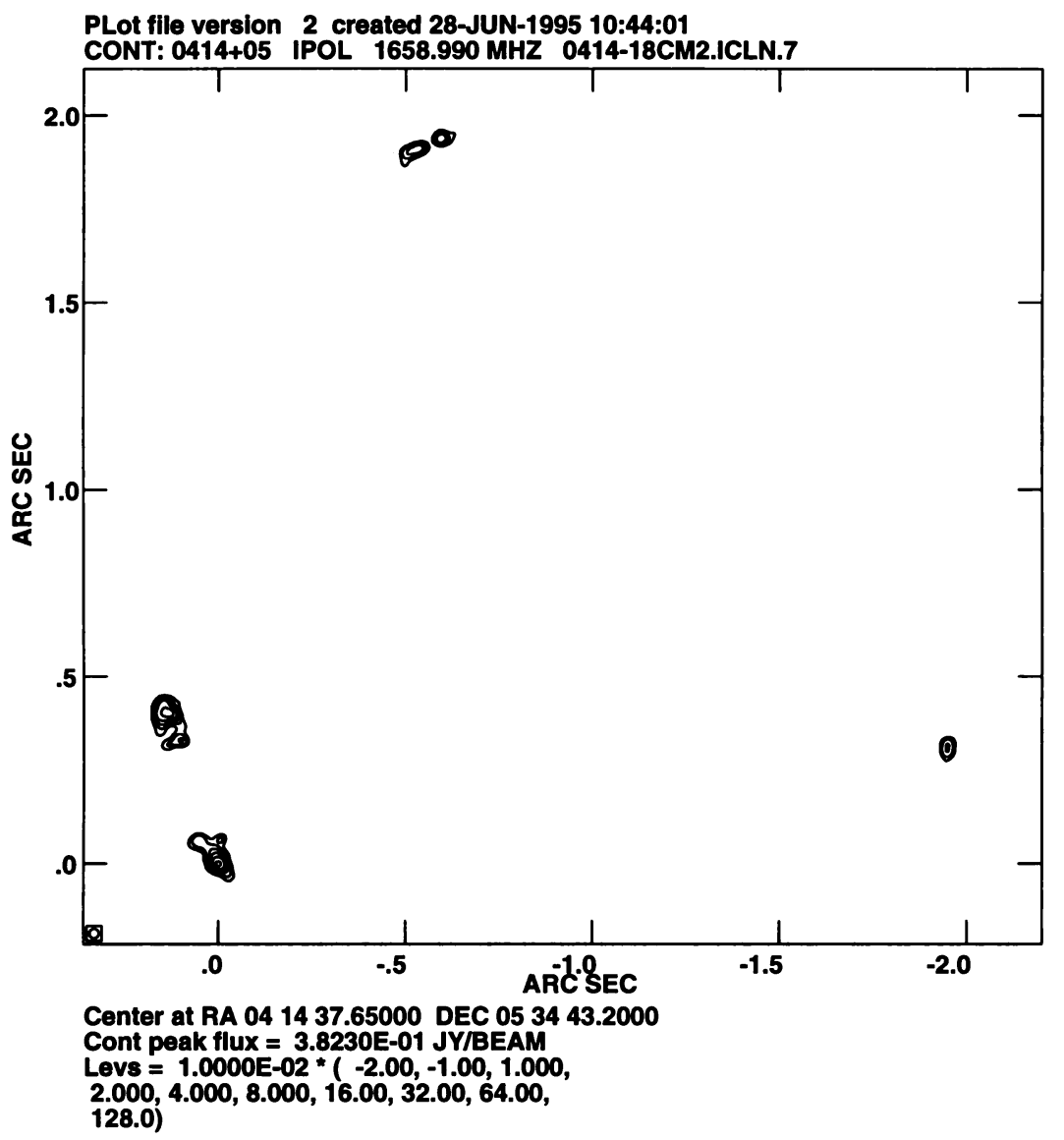

Figure 1. 1.7GHz EVN map of MG0414+0534.

nent has the brightest peak, and a jet-like extension pointing to the SE. The SW component also comprises a peak and a curving, jet-like extension pointing NW, as expected if it is the counterpart of that in the NE. Both components are resolved out on the long VLBI baselines between Europe and Hartebeesthoek, South Africa at this frequency. Jones (1994) presents $5 \mathrm{GHz}$ maps of the two compact components observed at two epochs. These also exhibit extensions along the jet directions, and apparent changes in their lengths between epochs; if this corresponds to real motion, it must be highly superluminal. These maps, and our own EVN $5 \mathrm{GHz}$ map, also show a prominent jet-like extension of the NE component in the NW direction, i.e. opposite to the $1.7 \mathrm{GHz}$ jet; no obvious counterpart to this feature is seen in the SW component, which has a higher peak brightness than the $\mathrm{NE}$ component at $5 \mathrm{GHz}$. Garrett et al. (these proceedings) report VLBA observations at $15 \mathrm{GHz}$ which show clearly this NW-pointing jet in the NE 

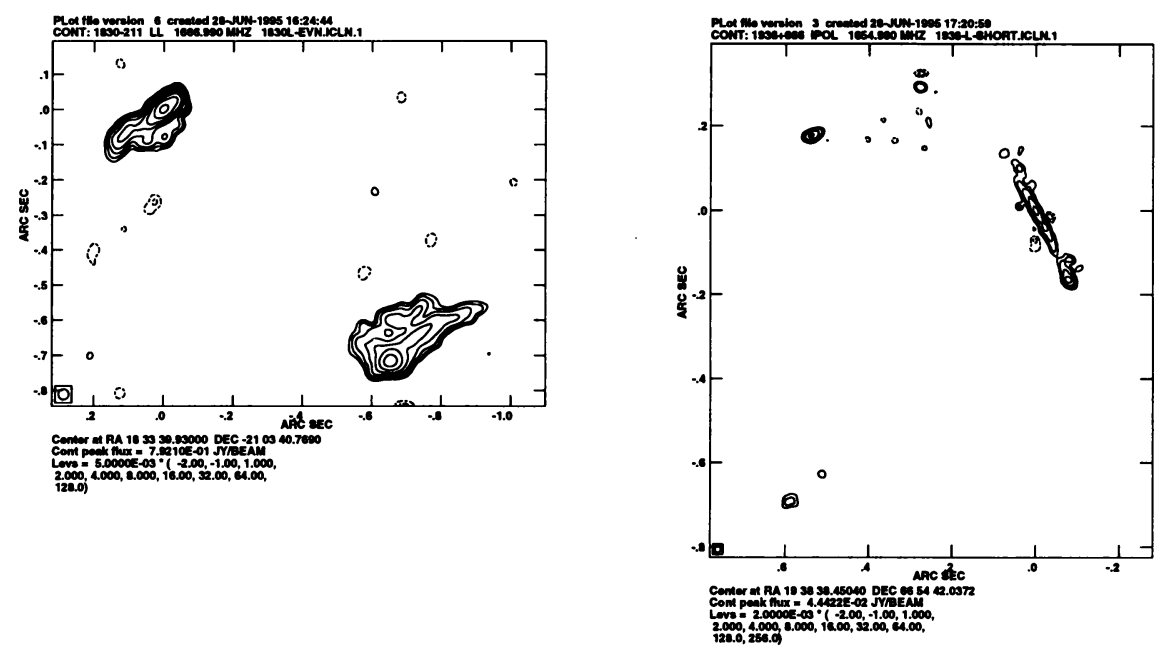

Figure 2. Left: $1.7 \mathrm{GHz}$ EVN map of PKS1830-211. Right: Global $1.7 \mathrm{GHz}$ map of $\mathrm{B} 1938+666$ using the short baselines, resolution 15mas.

component, but only a compact core in the SW. Jones et al. (these proceedings) speculate that interstellar scattering may cause the apparent size of the SW component to increase with wavelength. This may also explain our lack of detection on long baselines at $1.7 \mathrm{GHz}$.

B0218+357 This system consists of an Einstein ring and two image components, both of which show compact, core-jet structures (Patnaik et al. 1993, 1995). A detailed description of this, the smallest known system, is given in Porcas and Patnaik (these proceedings).

B1938+666 The MERLIN map (Patnaik 1994) shows a complex morphology, with a number of components spread around an 'arc', and a further 3 isolated point-like features. We have used our $1.7 \mathrm{GHz}$ global observations to make a 'low' resolution map (15mas resolution) using just the short baselines (Fig. 2, right) since the source was largely not detected on long baselines. All three point-like components are detected, as are point-like components at the extremes of the arc. However, the strongest feature is a long, thin, slightly curved portion of the arc. A cut along the arc at PA $24^{\circ}$ reveals at least two separate peaks along its length. At $5 \mathrm{GHz}$ we detect only the brightest feature in the arc.

B1422+231 This system is a 'quad' of overall size 1.3arcsec (Patnaik et al. 1992) with two equally bright radio images $A$ and $B(1: 1)$, and weaker images $C(1 / 2)$ and $D(1 / 30)$. Our global $1.7 \mathrm{GHz}$ observations detect all 4 components, and $\mathrm{A}, \mathrm{B}$ and $\mathrm{C}$ show faint extensions. Maps of all the images from our global $5 \mathrm{GHz}$ observations (1mas resolution) are shown in Figs. 3 and 4. Both A and B show elongated, core-jet like structures, 

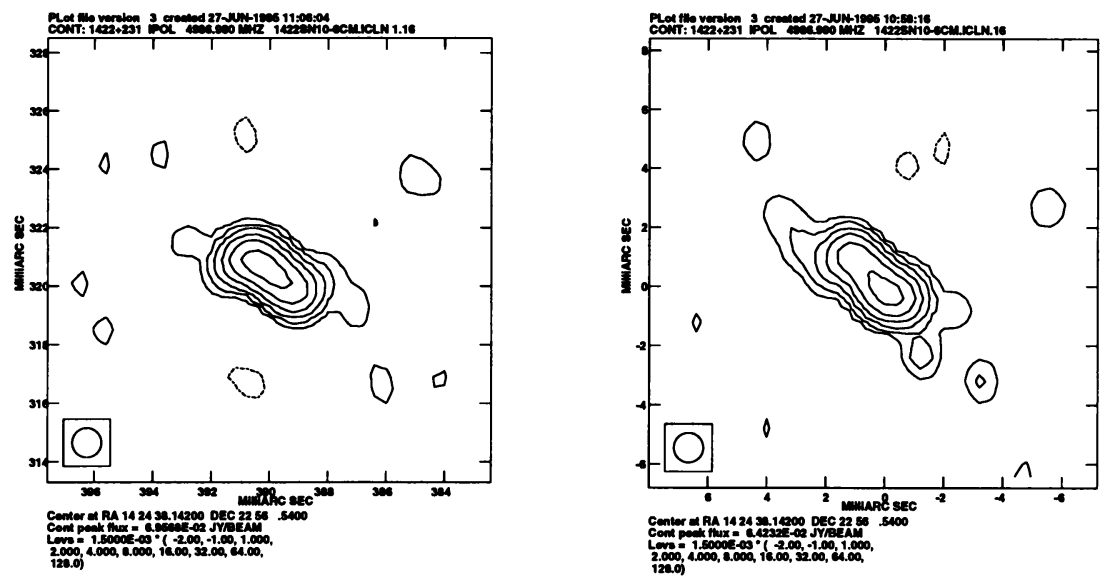

Figure 3. Global $5 \mathrm{GHz}$ map of $\mathrm{B} 1422+231 \mathrm{~A}($ left) and B (right), resolution 1mas.
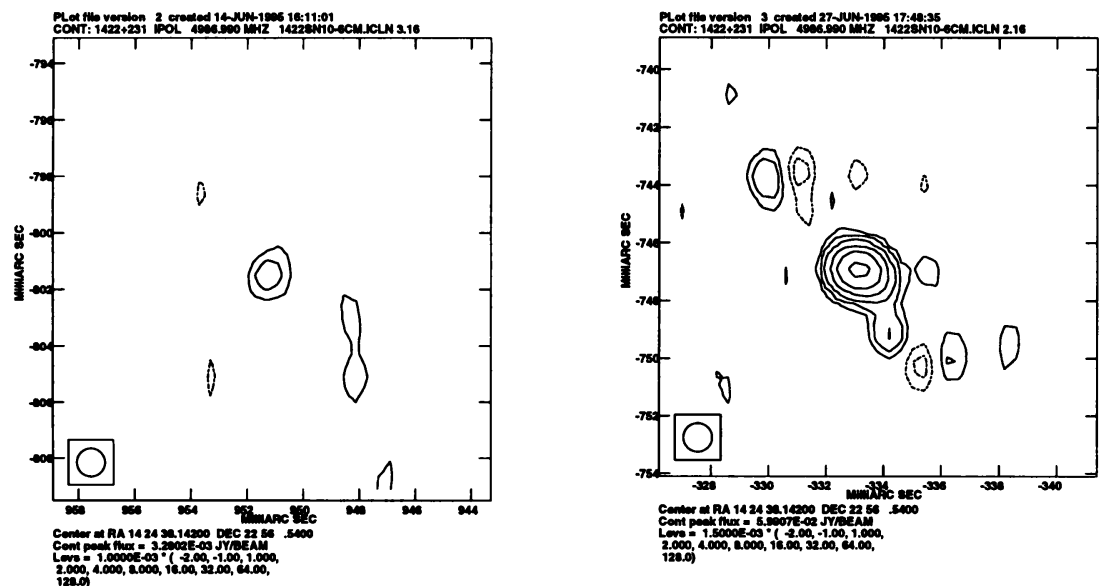

Figure 4. Global 5GHz map of B1422+231 D (left) and C (right), resolution 1mas.

indicating the expected tangential stretching. Component $\mathrm{C}$ also shows extended components. The brightness peaks of the $5 \mathrm{GHz}$ images coincide with the positions of $1.7 \mathrm{GHz}$ images.

\section{Conclusions}

Although the seven lens systems investigated with VLBI present a wealth of detail which can help constrain lens masses and examine the lensing process, it is clear that a number of puzzles remain. The interpretation of the mas radio structures of 2016+112 and PKS1830-211 is far from clear, and we still do not know to what extent magnification gradients across 
spectrally inhomogeneous images are affecting the observed radio image flux density ratios. The present radio searches will hopefully produce many new lens systems which can be studied at mas resolution using VLBI. Acknowledgements We wish to thank Michael Garrett and Ian Browne, who are our collaborators in some of the unpublished work presented here, and Jackie Hewitt, Dayton Jones and Josef Lehàr for communicating results in advance of publication. We also thank Sunita Nair for many interesting discussions.

\section{References}

Campbell, R.M., et al., 1994, ApJ, 426, 486

Campbell, R.M., Lehàr,J., Corey, B.E., Shapiro, I.I., \& Falco, E.E., 1995, AJ, submitted Garrett, M.A., 1990, Doctoral Thesis, U. Manchester, 90

Garrett, M.A., et al., 1994a, MNRAS, 270, 457

Garrett, M.A., Patnaik, A.R., \& Porcas, R.W., 1994b, in EVN/JIVE Symposium, Torun, ed. Kus et al., TRAO, 73

Gorenstein, M.V. et al., 1983, Science, 219, 54

Gorenstein, M.V. et al., 1984, ApJ, 287, 538

Gorenstein, M.V. et al., 1988, ApJ, 334, 42

Haschick, A.D., et al., 1981, ApJL, 243, L57

Heflin, M.B., Gorenstein, M.V., Lawrence, C.R. \& Burke, B.F., 1991, ApJ, 378, 519

Hewitt, J.N. et al., 1992, AJ, 104, 968

Jauncey, D.L. et al., 1991, Nature, 352, 132

Jones, D.L., 1994, in Proc. Compact Extragalactic Sources, ed. Zensus and Kellermann, NRAO, 135

Patnaik, A.R., et al., 1992, MNRAS, 259, 1p

Patnaik, A.R., 1994, in Gravitational Lenses in the Universe, Ed. Surdej et al., (Liège: Université de Liège), 311

Patnaik, A.R., et al., 1993, MNRAS, 261, 435

Patnaik, A.R., Porcas, R.W., \& Browne, I.W.A, 1995, MNRAS, 274, 5p

Porcas, R.W. et al., 1979, Nature, 282, 385

Porcas, R.W. et al., 1981, Nature, 289, 758

Porcas, R.W., 1994, in Compact Extragalactic Sources, ed. Zensus and Kellermann, NRAO, 125

Porcas, R.W. \& Patnaik, A.R., 1995, in 10th working Meeting on European VLBI for Geodesy and Astrometry, Matera (in press)

Rogers, A.E.E., 1989, in Gravitational Lenses, eds., Moran et al. (Dordrecht: Springer) 77 